\begin{tabular}{lcccr} 
T H E & A R C H I V E & O F & M E C H A N I C A L & E N G I N E E R I N G \\
\hline VOL. LX & 2013 & Number 2 \\
$\begin{array}{l}\text { 10.2478/meceng-2013-0015 } \\
\text { Key words: EMAT, nonlinear magnetoelasticity, nonlinear electroelasticity, electromechanical coupling, wave propa- } \\
\text { gation }\end{array}$
\end{tabular}

\title{
ON THE GENERAL GOVERNING EQUATIONS OF ELECTROMAGNETIC ACOUSTIC TRANSDUCERS
}

\begin{abstract}
In this paper, we present the general governing equations of electrodynamics and continuum mechanics that need to be considered while mathematically modelling the behaviour of electromagnetic acoustic transducers (EMATs). We consider the existence of finite deformations for soft materials and the possibility of electric currents, temperature gradients, and internal heat generation due to dissipation. Starting with Maxwell's equations of electromagnetism and balance laws of nonlinear elasticity, we present the governing equations and boundary conditions in incremental form in order to solve wave propagation problems of boundary value type.
\end{abstract}

\section{Introduction}

Electromagnetic acoustic transduction is a technique used to generate and detect mechanical waves in magnetoelastic conductors for the purpose of nondestructive testing. The devices, called electromagnetic acoustic transducers (EMATs) or electromagnetic acoustic receivers (EMARs), function by exploiting the coupling between electromagnetic and mechanical effects in a deformable continuum. The transducer mechanism (as shown in the schematic in Figure 1) consists of a current-carrying coil suspended on the specimen under test which is magnetized by a large static magnetic field. An AC current is passed through the coil which generates a time-varying magnetic field in the specimen. This, on interaction with the static magnetic field, leads to an electromagnetic body force that causes the generation of mechanical waves in the specimen. In the case of EMARs, the existing mechanical waves in the specimen, on interaction with the static magnetic field, produce time-varying magnetic field in the vicinity. This generates an AC

* Chair of Applied Mechanics, University of Erlangen-Nuremberg, Egerlandstrasse 5, 91058 Erlangen, Germany; E-mail: prashant.saxena@ltm.uni-erlangen.de 
current in the current-carrying coil which can be measured to analyze the mechanical waves in the specimen. Thus, it is very important to understand the exact coupling between mechanical and electromagnetic fields for an accurate mathematical modelling of the process.

Several orientations of the specimen and the coil for EMATs have been studied in the recent literature. Much of the work (including [1]) is based, among other literature, on the papers by Ludwig et al. [2], Ogi [3], and Thompson [4] on the theory and numerical simulation of EMATs. Recently, Shapoorabadi et al. [5] have presented the governing equations of EMATs using a formulation involving the magnetic vector potential. The list of references is by no means exhaustive and there are many more papers and theses on this subject.

However, the existing theory of EMATs is, in our view, incomplete in some respects. Many theoretical models have been developed and experimental results been obtained for only a sinusoidal steady state of current and displacement (such as [4]). A large volume of literature assumes linear elastic deformations in the specimen and a linear coupling between the electromagnetic and mechanical phenomena (such as [2], [5]). This is, however, not the case in reality with the coupling being nonlinear, as has been presented in the classical texts by Pao [6], Eringen and Maugin [7], and in the recent paper by Maugin [8]. Moreover, with the development of soft polymeric electroand magnetoelastic smart materials ([9], [10]) in the recent years, one needs to take into account the possibility of finite deformations. In some materials, heat generation caused by electric currents may lead to significant changes in magneto-elastic properties, hence these effects also need to be taken into account.

In this paper, we present the equations that govern wave propagation in a finitely deformed solid in the presence of electromagnetic fields. On the existing finite deformation, magnetization, and electric polarization, time dependent small increments in the electric, magnetic, temperature, and deformation fields are allowed. We provide a general constitutive formulation based on a free energy function that depends on the initial deformation, underlying electromagnetic fields, and temperature. This is based on a generalization of the constitutive formulations of electroelasticity and magnetoelasticity provided by Dorfmann and Ogden [11], [12]. The same formulation of magnetoelasticity has been used by Destrade and Ogden [13], and author and co-workers [14], [15] to study some wave propagation problems in magnetoelasticity.

In Section 2, we recapitulate the basic governing equations of electromagnetism in continua and the laws of thermodynamics as given by Pao [6] and Eringen and Maugin [7]. These are used to derive constitutive relations from a free energy function. We then allow for small time-dependent incre- 
ments in Section 4 to study wave propagation. Several 'moduli' tensors are also introduced in this section to quantify the couplings in elastic, electric, magnetic, and temperature fields. It is expected that the general equations provided here will be solved using numerical schemes such as FEM for specific boundary problems related to EMATs.

\section{Basic equations}

The undeformed stress-free reference configuration of a continuous elastic body is denoted by $\mathcal{B}_{r}$ and its boundary by $\partial \mathcal{B}_{r}$. Let $\mathcal{B}_{t}$, the current configuration, be the region occupied by the body at time $t$ and $\partial \mathcal{B}_{t}$ its boundary. The material points of body are identified by the position vector $\mathbf{X}$ in $\mathcal{B}_{r}$ which becomes the position vector $\mathbf{x}$ in $\mathcal{B}_{t}$.

\subsection{Kinematics}

The time-dependent deformation (or motion) of the body is described by an invertible mapping $\chi$ that maps points from $\mathcal{B}_{r}$ to points in $\mathcal{B}_{t}$ such that $\mathbf{x}=\chi(\mathbf{X}, t)$. The function $\chi$ and its inverse are assumed to be sufficiently regular in space and time. The velocity $\mathbf{v}$ and acceleration $\mathbf{a}$ of a material particle at $\mathbf{X}$ are defined by

$$
\mathbf{v}(\mathbf{x}, t)=\mathbf{x}_{, t}=\frac{\partial}{\partial t} \chi(\mathbf{X}, t), \quad \mathbf{a}(\mathbf{x}, t)=\mathbf{v}_{, t}=\mathbf{x}_{, t t}=\frac{\partial^{2}}{\partial t^{2}} \chi(\mathbf{X}, t),
$$

where the subscript $t$ following a comma denotes the material time derivative. In this paper; Grad, Div, and Curl denote the standard differential operators in the reference configuration while grad, div, and curl denote the same in the current configuration. The deformation gradient tensor is defined as $\mathbf{F}=\operatorname{Grad} \chi(\mathbf{X}, t)$ and its determinant is denoted by $J=\operatorname{det} \mathbf{F}$, with $J>0$. Associated with $\mathbf{F}$ is the right Cauchy-Green tensor $\mathbf{c}=\mathbf{F}^{\mathrm{T}} \mathbf{F}$. For an incompressible material the constraint $J=\operatorname{det} \mathbf{F}=1$, needs to be satisfied.

\subsection{Maxwell's equations}

The well-known Maxwell's equations governing electromagnetic fields in a deformable continua (the region $\mathcal{B}_{t}$ in Figure 1) are given by the DipoleCurrent Circuit Model of Pao [6] as

$$
\begin{array}{r}
\operatorname{div} \mathbf{B}=0, \quad \operatorname{curl} \mathbf{E}+\frac{\partial \mathbf{B}}{\partial t}=0, \quad \operatorname{div}\left(\varepsilon_{0} \mathbf{E}+\mathbf{P}\right)=\rho_{e}, \\
\operatorname{curl}\left(\frac{1}{\mu_{0}} \mathbf{B}-\mathbf{M}\right)=\mathbf{J}+\frac{\partial}{\partial t}\left(\varepsilon_{0} \mathbf{E}+\mathbf{P}\right),
\end{array}
$$




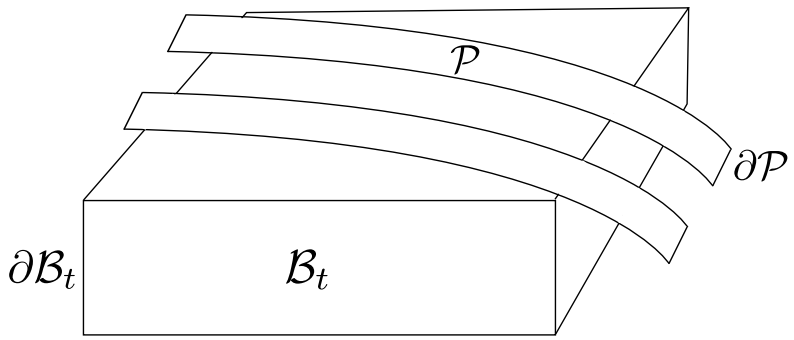

Fig. 1. A generic EMAT configuration. $\mathcal{B}_{t}$ and $\mathcal{P}$ are the regions occupied by the continuum material and a wire of the current-carrying coil, respectively. The corresponding boundaries are denoted by $\partial \mathcal{B}_{t}$ and $\partial \mathcal{P}$

where $\mathbf{B}$ is the magnetic induction vector, $\mathbf{E}$ is the electric field vector, $\mathbf{M}$ and $\mathbf{P}$ are, respectively, the magnetization and electric polarization of the continuum; $\mathbf{J}$ is the electric current density, $\rho_{e}$ is the electric charge density, $\varepsilon_{0}$ and $\mu_{0}$ are the electrical pemittivity and magnetic permeability of the vacuum respectively. We have used the following field relations in the above equations

$$
\mathbf{D}=\varepsilon_{0} \mathbf{E}+\mathbf{P}, \quad \mathbf{B}=\mu_{0}(\mathbf{H}+\mathbf{M}) .
$$

All the electromagnetic quantities above are defined in the current (Eulerian) configuration. They can also be expressed in the reference configuration (Lagrangian form) given by

$$
\begin{array}{r}
\mathbf{B}_{l}=J \mathbf{F}^{-1} \mathbf{B}, \quad \mathbf{P}_{l}=J \mathbf{F}^{-1} \mathbf{P}, \quad \mathbf{E}_{l}=\mathbf{F}^{\mathrm{T}} \mathbf{E}, \quad \mathbf{M}_{l}=\mathbf{F}^{\mathrm{T}} \mathbf{M}, \\
\mathbf{J}_{\mathrm{E}}=J \mathbf{F}^{-1}\left(\mathbf{J}-\rho_{\mathrm{e}} \mathbf{v}\right), \quad \rho_{\mathrm{E}}=J \rho_{\mathrm{e}},
\end{array}
$$

using which the Maxwell's equations can be written in Lagrangian form as

$$
\operatorname{Div} \mathbf{B}_{l}=0, \quad \operatorname{Curl}\left(\mathbf{E}_{l}+\mathbf{V} \times \mathbf{B}_{l}\right)=-\mathbf{B}_{l, t}, \quad \varepsilon_{0} \operatorname{Div}\left(J \mathbf{c}^{-1} \mathbf{E}_{l}\right)=\rho_{\mathrm{E}}+\operatorname{Div} \mathbf{P}_{l},
$$

$$
\begin{aligned}
\operatorname{Curl}\left(\frac{J^{-1}}{\mu_{0}} \mathbf{c B}_{l}-\varepsilon_{0} \mathbf{V} \times\left(J \mathbf{c}^{-1} \mathbf{E}_{l}\right)\right) & -\varepsilon_{0}\left(J \mathbf{c}^{-1} \mathbf{E}_{l}\right)_{, t} \\
& =\mathbf{P}_{l, t}+\operatorname{Curl}\left(\mathbf{M}_{l}+\mathbf{V} \times \mathbf{P}_{l}\right)+\mathbf{J}_{E} .
\end{aligned}
$$

In the current-carrying coil of rigid material (region $\mathcal{P}$ in Figure 1), the governing equations for the electromagnetic fields are

$$
\operatorname{div} \mathbf{B}=0, \quad \operatorname{curl} \mathbf{E}+\frac{\partial \mathbf{B}}{\partial t}=0, \quad \frac{1}{\mu_{0} \mu_{r}} \operatorname{curl} \mathbf{B}=\varepsilon_{0} \varepsilon_{r} \frac{\partial \mathbf{E}}{\partial t}+\mathbf{J}, \quad \operatorname{div} \mathbf{E}=0,
$$

where the constants $\varepsilon_{r}$ and $\mu_{r}$ are the relative electric permittivity and the relative magnetic permeability of the current-carrying coil, respectively. 
Outside the material, in a vacuum, the governing equations for electromagnetic fields are

$$
\operatorname{div} \mathbf{B}^{*}=0, \quad \operatorname{curl} \mathbf{E}^{*}+\frac{\partial \mathbf{B}^{*}}{\partial t}=\mathbf{0}, \quad \operatorname{div} \mathbf{E}^{*}=0, \quad \operatorname{curl} \mathbf{B}^{*}-\varepsilon_{0} \mu_{0} \frac{\partial \mathbf{E}^{*}}{\partial t}=\mathbf{0},
$$

where we denote a physical quantity in vacuum by a superscript *

At the boundary $\partial \mathcal{B}_{r}$, the following conditions need to be satisfied (see, for example, [15])

$$
\begin{aligned}
& \mathbf{N} \times\left(\mathbf{E}_{l}+\mathbf{V} \times \mathbf{B}_{l}-\mathbf{F}^{\mathrm{T}} \mathbf{E}^{*}\right)=\mathbf{0} \\
& \mathbf{N} \cdot\left(\mathbf{B}_{l}-J \mathbf{F}^{-1} \mathbf{B}^{*}\right)=0 \\
& \mathbf{N} \cdot\left\{\varepsilon_{0} J \mathbf{c}^{-1}\left(\mathbf{E}_{l}-\mathbf{F}^{\mathrm{T}} \mathbf{E}^{*}\right)+\mathbf{P}_{l}\right\}=\sigma_{E}, \\
& \mathbf{N} \times\left(J^{-1} \mu_{0}^{-1} \mathbf{c B}_{l}-\mathbf{M}_{l}-\mathbf{V} \times\left(\varepsilon_{0} J \mathbf{c}^{-1} \mathbf{E}_{l}+\mathbf{P}_{l}\right)-\mu_{0}^{-1} \mathbf{F}^{\mathrm{T}} \mathbf{B}^{*}\right)=\mathbf{K}_{l}-\sigma_{E} \mathbf{V}_{\mathrm{s}},
\end{aligned}
$$

where $\mathbf{N}$ is the unit normal to the boundary, $\mathbf{V}=\mathbf{F}^{-1} \mathbf{v}$ is the particle velocity, $\mathbf{V}_{\mathrm{s}}$ is the value of $\mathbf{V}$ at the boundary, $\sigma_{E}$ is the surface electric charge density, and $\mathbf{K}_{l}$ is Lagrangian description of the surface current density. We note that $\mathbf{F}$ is not defined outside the material and the values of $\mathbf{F}$ and $J$ calculated on the boundary are used in the above equations.

The boundary conditions are simpler at $\partial \mathcal{P}_{t}$ and given by

$$
\begin{aligned}
& \mathbf{n} \times\left(\mathbf{E}-\mathbf{E}^{*}\right)=\mathbf{0}, \quad \mathbf{n} \cdot\left(\varepsilon_{r} \mathbf{E}-\mathbf{E}^{*}\right)=0, \\
& \mathbf{n} \times\left(\frac{\mathbf{B}}{\mu_{r}}-\mathbf{B}^{*}\right)=\mathbf{K}, \quad \mathbf{n} \cdot\left(\mathbf{B}-\mathbf{B}^{*}\right)=0 .
\end{aligned}
$$

\subsection{Mechanical balance laws}

Balance of linear momentum, in the absence of a mechanical body force, is given by

$$
\operatorname{div} \boldsymbol{\tau}+\mathbf{f}_{e}=\rho \mathbf{a}
$$

where $\tau$ is the Cauchy stress tensor, $\rho$ is the mass density, and the electromagnetic body force as given in [6] is

$$
\mathbf{f}_{e}=\rho_{e} \mathbf{E}+\mathbf{J} \times \mathbf{B}+(\operatorname{grad} \mathbf{E})^{\mathrm{T}} \mathbf{P}+(\operatorname{grad} \mathbf{B})^{\mathrm{T}} \mathbf{M}+\frac{\partial}{\partial t}(\mathbf{P} \times \mathbf{B})+\operatorname{div}[\mathbf{v} \otimes(\mathbf{P} \times \mathbf{B})]
$$

Balance of angular momentum gives

$$
\boldsymbol{\varepsilon} \tau+\mathbf{L}_{e}=\mathbf{0}, \quad \mathbf{L}_{e}=\mathbf{P} \times \mathbf{E}+(\mathbf{M}+\mathbf{v} \times \mathbf{P}) \times \mathbf{B},
$$


where $\boldsymbol{\varepsilon}$ is the third order permutation tensor with components $\varepsilon_{i j k}$ and $(\boldsymbol{\varepsilon} \tau)_{i}=\varepsilon_{i j k} \tau_{j k}$ and $\mathbf{L}_{e}$ is the electromagnetic body couple vector. The above balance equations can be written in Lagrangian form using the nominal stress tensor $\mathbf{T}=J \mathbf{F}^{-1} \boldsymbol{\tau}$ as

$$
\operatorname{Div} \mathbf{T}+J \mathbf{f}_{E}=\rho_{r} \mathbf{a}, \quad \boldsymbol{\varepsilon}(\mathbf{F T})+J \mathbf{L}_{E}=\mathbf{0},
$$

where $\mathbf{f}_{E}$ and $\mathbf{L}_{E}$ are Lagrangian counterparts of the corresponding vectors and are given by

$$
\begin{array}{r}
\mathbf{f}_{E}=J^{-1} \rho_{\mathrm{E}} \mathbf{F}^{-\mathrm{T}} \mathbf{E}_{l}+J^{-2}\left(\mathbf{F} \mathbf{J}_{l}\right) \times\left(\mathbf{F} \mathbf{B}_{l}\right)+\mathbf{F}^{-\mathrm{T}}\left[\operatorname{Grad}\left(\mathbf{F}^{-\mathrm{T}} \mathbf{E}_{l}\right)\right]^{\mathrm{T}}\left(J^{-1} \mathbf{F} \mathbf{P}_{l}\right) \\
+\mathbf{F}^{-\mathrm{T}}\left[\operatorname{Grad}\left(J^{-1} \mathbf{F} \mathbf{B}_{l}\right)\right]^{\mathrm{T}}\left(\mathbf{F}^{-\mathrm{T}} \mathbf{M}_{l}\right)+\frac{\partial}{\partial t}\left[J^{-2}\left(\mathbf{F} \mathbf{P}_{l}\right) \times\left(\mathbf{F} \mathbf{B}_{l}\right)\right] \\
+J^{-1} \operatorname{Div}\left[J^{-1} \mathbf{V} \otimes\left\{\left(\mathbf{F} \mathbf{P}_{l}\right) \times\left(\mathbf{F} \mathbf{B}_{l}\right)\right\}\right], \\
\mathbf{L}_{E}=J^{-1}\left(\mathbf{F} \mathbf{P}_{l}\right) \times\left(\mathbf{F}^{-\mathrm{T}} \mathbf{E}_{l}\right)+J^{-1}\left(\mathbf{F}^{-\mathrm{T}} \mathbf{M}_{e l}\right) \times\left(\mathbf{F} \mathbf{B}_{l}\right) .
\end{array}
$$

On any part of the boundary where the traction is prescribed, the boundary condition may be given as

$$
\mathbf{T}^{\mathrm{T}} \mathbf{N}=\mathbf{t}_{\mathrm{A}},
$$

where $\mathbf{t}_{\mathrm{A}}$ is the Lagrangian representation of the traction force.

\subsection{Energy balance laws and Constitutive relations}

First law of thermodynamics gives the balance of energy as

$$
\rho \frac{d U}{d t}=\boldsymbol{\tau}: \operatorname{grad} \mathbf{v}-\operatorname{div} \mathbf{q}+q+w_{e},
$$

where $U$ is the internal energy, $\mathbf{q}$ is the heat flux at the surface, $q$ is the volumetric heat generation, the symbol : denotes a scalar product between two second order tensors given in component form as $\tau: \Gamma=\tau_{i j} \Gamma_{j i}$, and $w_{e}$ is the electromagnetic power (see, for example, Pao [6]) given by

$$
w_{e}=\mathbf{J}_{e} \cdot \mathbf{E}_{e}+\rho \frac{d}{d t}\left(\frac{\mathbf{P}}{\rho}\right) \cdot \mathbf{E}_{e}-\mathbf{M}_{e} \cdot \frac{d \mathbf{B}}{d t},
$$

where for a dynamic problem we have defined the effective field variables as

$$
\mathbf{J}_{e}=\mathbf{J}-\rho_{e} \mathbf{v}, \quad \mathbf{E}_{e}=\mathbf{E}+\mathbf{v} \times \mathbf{B}, \quad \mathbf{M}_{e}=\mathbf{M}+\mathbf{v} \times \mathbf{P} .
$$


Let $\vartheta$ be the absolute temperature, then we can rewrite (22) as

$$
\rho c_{p} \frac{\partial \vartheta}{\partial t}=q+w_{e}+\tau: \operatorname{grad} \mathbf{v}-\operatorname{div} \mathbf{q}
$$

Here $c_{p}$ is the specific heat capacity. On defining the pullback versions of the physical quantities

$$
\mathbf{q}_{l}=J \mathbf{F}^{-1} \mathbf{q}, \quad q_{l}=J q, \quad w_{E}=J w_{e}, \quad \vartheta_{l}=J \vartheta,
$$

the above equation can be written in Lagrangian form as

$$
\rho_{r} c_{p} \frac{\partial}{\partial t}\left(J^{-1} \vartheta_{l}\right)=\mathbf{T}: \operatorname{Grad}(\mathbf{F V})+q_{l}+w_{E}-\operatorname{Div} \mathbf{q}_{l} .
$$

Here, the Lagrangian form of electromagnetic power is given as

$$
\begin{array}{r}
w_{E}=\left(\mathbf{F} \mathbf{J}_{e l}\right) \cdot\left(\mathbf{F}^{-\mathrm{T}} \mathbf{E}_{e l}\right)+\rho_{r}\left[\frac{\partial}{\partial t}\left(\frac{\mathbf{F} \mathbf{P}_{l}}{\rho_{r}}\right)+\operatorname{Grad}\left(\frac{\mathbf{F} \mathbf{P}_{l}}{\rho_{r}}\right) \mathbf{V}\right] \cdot\left(\mathbf{F}^{-\mathrm{T}} \mathbf{E}_{e l}\right) \\
-J \mathbf{F}^{-\mathrm{T}} \mathbf{M}_{e l} \cdot\left[\frac{\partial}{\partial t}\left(J^{-1} \mathbf{F} \mathbf{B}_{l}\right)+\operatorname{Grad}\left(J^{-1} \mathbf{F} \mathbf{B}_{l}\right) \mathbf{V}\right] .
\end{array}
$$

If $S$ is the entropy density, then the second law of thermodynamics is given by the Clausius-Duhem inequality as

$$
\rho \frac{d S}{d t}+\operatorname{div}\left(\frac{\mathbf{q}}{\vartheta}\right)-\frac{q}{\vartheta} \geq 0 .
$$

Substituting equation (22) into (29) and following the standard ColemanNoll procedure (see, for e.g., [16]), we arrive at the constitutive relations

$$
\mathbf{T}=\frac{\partial \Phi}{\partial \mathbf{F}}, \quad \mathbf{P}_{l}=-\frac{\partial \Phi}{\partial \mathbf{E}_{e l}}, \quad \mathbf{M}_{e l}=-\frac{\partial \Phi}{\partial \mathbf{B}_{l}},
$$

where $\Phi$ is the free energy per unit volume related to $U$ (see, for example, [15] for details) as

$$
\Phi\left(\mathbf{F}, \mathbf{E}_{e l}, \mathbf{B}_{l}, \vartheta_{l}\right)=\rho_{r}\left(U\left(\mathbf{F}, \mathbf{E}_{e}, \mathbf{B}, \vartheta\right)-\vartheta S-\mathbf{E}_{e} \cdot \frac{\mathbf{P}}{\rho}\right) .
$$

We note that in the case of an incompressible material, we have an additional constraint $J=1$ and the constitutive law for stress is modified to

$$
\mathbf{T}=\frac{\partial \Phi}{\partial \mathbf{F}}-p \mathbf{F}^{-1}
$$


$p$ being the Lagrange multiplier associated with the constraint of incompressibility.

We assume the constitutive laws governing heat flow and electric current flow to be given by the Fourier's law and the Ohm's law, respectively, as

$$
\mathbf{q}=-\kappa \operatorname{grad} \vartheta, \quad \mathbf{J}=\boldsymbol{\xi} \mathbf{E},
$$

where $\boldsymbol{\kappa}$ and $\boldsymbol{\xi}$ are positive-definite symmetric second order tensors quantifying the thermal conductivity and the electrical conductivity, respectively. We require tensorial values for the conductivities since in general for anisotropic materials, the conductivity depends on the direction of heat flow or electric current. For isotropic materials, these assume the values $\boldsymbol{\kappa}=\kappa \mathbf{I}$ and $\boldsymbol{\xi}=\boldsymbol{\xi} \mathbf{I}$. The above equations can be written in Lagrangian form as

$$
\mathbf{q}_{l}=-J \mathbf{F}^{-1} \boldsymbol{\kappa} \mathbf{F}^{-\mathrm{T}} \operatorname{Grad}\left(J^{-1} \vartheta_{l}\right), \quad \mathbf{J}_{l}=J \mathbf{F}^{-1} \boldsymbol{\xi} \mathbf{F}^{-\mathrm{T}} \mathbf{E}_{l} .
$$

\section{Incremental equations}

On the initial motion and underlying electromagnetic fields, we consider an incremental mechanical motion $\mathbf{u}(\mathbf{x}, t)$, and increments in electromagnetic fields which are denoted by a superposed dot. We emphasize here the departure from convention in using a superposed dot to denote an increment rather than a time-derivative for the sake of brevity.

The incremented forms of the Lagrangian Maxwell's equations (6) and (7) are given as

$$
\begin{gathered}
\operatorname{Div} \dot{\mathbf{B}}_{l}=0, \quad \operatorname{Curl} \dot{\mathbf{E}}_{e l}=-\dot{\mathbf{B}}_{l, t}, \\
\varepsilon_{0} \operatorname{Div}\left[J(\operatorname{div} \mathbf{u}) \mathbf{c}^{-1} \mathbf{E}_{l}+J \mathbf{c}^{-1} \dot{\mathbf{E}}_{l}-J \mathbf{F}^{-1}\left(\mathbf{L}+\mathbf{L}^{\mathrm{T}}\right) \mathbf{F}^{-\mathrm{T}} \mathbf{E}_{l}\right]=\dot{\rho}_{\mathrm{E}}+\operatorname{Div} \dot{\mathbf{P}}_{l}, \\
\mu_{0}^{-1} \operatorname{Curl}\left[J^{-1}\left\{(\operatorname{div} \mathbf{u}) \mathbf{c} \mathbf{B}_{l}+2 \mathbf{F}^{\mathrm{T}} \mathbf{L F} \mathbf{B}_{l}+\mathbf{c} \dot{\mathbf{B}}_{l}\right\}\right]-\varepsilon_{0} \operatorname{Curl}\left[\dot{\mathbf{V}} \times\left(J \mathbf{c}^{-1} \mathbf{E}_{l}\right)\right. \\
\left.+\mathbf{V} \times\left\{J(\operatorname{div} \mathbf{u}) \mathbf{c}^{-1} \mathbf{E}_{l}+J \mathbf{c}^{-1} \dot{\mathbf{E}}_{l}-J \mathbf{F}^{-1}\left(\mathbf{L}+\mathbf{L}^{\mathrm{T}}\right) \mathbf{F}^{-\mathrm{T}} \mathbf{E}_{l}\right\}\right] \\
-\varepsilon_{0}\left[J\left\{J(\operatorname{div} \mathbf{u}) \mathbf{c}^{-1} \mathbf{E}_{l}+J \mathbf{c}^{-1} \dot{\mathbf{E}}_{l}-J \mathbf{F}^{-1}\left(\mathbf{L}+\mathbf{L}^{\mathrm{T}}\right) \mathbf{F}^{-\mathrm{T}} \mathbf{E}_{l}\right\}\right]_{, t} \\
=\dot{\mathbf{P}}_{l, t}+\operatorname{Curl} \dot{\mathbf{M}}_{e l}+\dot{\mathbf{J}}_{\mathrm{E}} .
\end{gathered}
$$

The incremented Lagrangian quantities are 'pushed forward' to the Eulerian configuration and denoted by a subscript ' 0 ' after ' $l$ ' or ' $E$ '. The pushforward relations for the incremented fields are given (similar to (5)) as

$$
\begin{array}{r}
\dot{\mathbf{B}}_{l 0}=J^{-1} \mathbf{F} \dot{\mathbf{B}}_{l}, \quad \dot{\mathbf{P}}_{l 0}=J^{-1} \dot{\mathbf{F}}_{l}, \quad \dot{\mathbf{M}}_{e l 0}=\mathbf{F}^{-\mathrm{T}} \dot{\mathbf{M}}_{e}, \\
\dot{\mathbf{E}}_{l 0}=\mathbf{F}^{-\mathrm{T}} \dot{\mathbf{E}}_{l} \quad \dot{\rho}_{E 0}=J^{-1} \dot{\rho}_{E},
\end{array}
$$


using which we can write the Eulerian form of the above incremental equations as

$$
\begin{gathered}
\operatorname{div} \dot{\mathbf{B}}_{l 0}=0, \quad \operatorname{curl} \dot{\mathbf{E}}_{e l 0}=[\mathbf{\Gamma}-(\operatorname{div} \mathbf{v}) \mathbf{I}] \dot{\mathbf{B}}_{l 0}-\dot{\mathbf{B}}_{l 0, t}, \\
\varepsilon_{0} \operatorname{div} \hat{\mathbf{E}}=\dot{\rho}_{\mathrm{E} 0}+\operatorname{div} \dot{\mathbf{P}}_{l 0}, \\
\mu_{0}^{-1} \operatorname{curl}\left[\{(1+\operatorname{div} \mathbf{u}) \mathbf{I}+2 \mathbf{L}\} \dot{\mathbf{B}}_{l 0}\right]-\varepsilon_{0} \operatorname{curl}\left(\mathbf{u}_{, t} \times \mathbf{E}+\mathbf{v} \times \hat{\mathbf{E}}\right)-\varepsilon_{0} \hat{\mathbf{E}}_{, t} \\
=\operatorname{curl} \dot{\mathbf{M}}_{e l 0}+\dot{\mathbf{P}}_{l 0, t}+[(\operatorname{div} \mathbf{v}) \mathbf{I}-\mathbf{\Gamma}] \dot{\mathbf{P}}_{l 0}+\dot{\mathbf{J}}_{\mathrm{E} 0,}
\end{gathered}
$$

where

$$
\begin{aligned}
\hat{\mathbf{E}} & =\dot{\mathbf{E}}_{l 0}+(\operatorname{div} \mathbf{u}) \mathbf{E}-\left(\mathbf{L}+\mathbf{L}^{\mathrm{T}}\right) \mathbf{E}, \\
\dot{\mathbf{E}}_{e l 0} & =\mathbf{F}^{-\mathrm{T}} \dot{\mathbf{E}}_{e l}=\dot{\mathbf{E}}_{l 0}+\mathbf{v} \times \dot{\mathbf{B}}_{l 0}+\left(\mathbf{u}_{, t}-\mathbf{L v}\right) \times \mathbf{B}, \\
\dot{\mathbf{J}}_{E 0} & =J^{-1} \mathbf{F} \dot{\mathbf{J}}_{E}=\xi \hat{\mathbf{E}}-\dot{\rho}_{\mathrm{E} 0} \mathbf{v}-\rho_{\mathrm{e}}\left(\mathbf{u}_{, t}-\mathbf{L v}\right) .
\end{aligned}
$$

The incremented momentum and angular momentum balance equations (18) are given as

$$
\begin{gathered}
\operatorname{Div} \dot{\mathbf{T}}+J(\operatorname{div} \mathbf{u}) \mathbf{f}_{E}+J \dot{\mathbf{f}}_{E}=\rho_{r} \dot{\mathbf{a}}, \\
\boldsymbol{\varepsilon}(\mathbf{L F T}+\mathbf{F} \dot{\mathbf{T}})+J(\operatorname{div} \mathbf{u}) \mathbf{L}_{E}+J \dot{\mathbf{L}}_{E}=\mathbf{0} .
\end{gathered}
$$

where the increments in electromagnetic body force and moment are given by

$$
\begin{aligned}
& \dot{\mathbf{f}}_{E}=J^{-1}\left\{-(\operatorname{div} \mathbf{u}) \rho_{\mathrm{E}} \mathbf{F}^{-\mathrm{T}} \mathbf{E}_{l}+\dot{\rho}_{\mathrm{E}} \mathbf{F}^{-\mathrm{T}} \mathbf{E}_{l}-\rho_{\mathrm{E}} \mathbf{L}^{\mathrm{T}} \mathbf{F}^{-\mathrm{T}} \mathbf{E}_{l}+\rho_{\mathrm{E}} \mathbf{F}^{-\mathrm{T}} \dot{\mathbf{E}}_{l}\right\} \\
& -J^{-1} \mathbf{L}^{\mathrm{T}} \mathbf{F}^{-\mathrm{T}}\left[\operatorname{Grad}\left(\mathbf{F}^{-\mathrm{T}} \mathbf{E}_{l}\right)\right]^{\mathrm{T}}\left(\mathbf{F} \mathbf{P}_{l}\right)+J^{-1} \mathbf{F}^{-\mathrm{T}}\left[\operatorname{Grad}\left(-\mathbf{L}^{\mathrm{T}} \mathbf{F}^{-\mathrm{T}} \mathbf{E}_{l}+\mathbf{F}^{-\mathrm{T}} \dot{\mathbf{E}}_{l}\right)\right]^{\mathrm{T}}\left(\mathbf{F} \mathbf{P}_{l}\right) \\
& +J^{-1} \mathbf{F}^{-\mathrm{T}}\left[\operatorname{Grad}\left(\mathbf{F}^{-\mathrm{T}} \mathbf{E}_{l}\right)\right]^{\mathrm{T}}\left[-(\operatorname{div} \mathbf{u}) \mathbf{F} \mathbf{P}_{l}+\mathbf{L F} \mathbf{P}_{l}+\mathbf{F} \dot{\mathbf{P}}_{l}\right] \\
& +2 J^{-2}(\operatorname{div} \mathbf{u})\left(\mathbf{F} \mathbf{J}_{l}\right) \times\left(\mathbf{F B} \mathbf{B}_{l}\right)+J^{-2}\left(\mathbf{L F} \mathbf{J}_{l}+\mathbf{F} \mathbf{J}_{l}\right) \times\left(\mathbf{F} \mathbf{B}_{l}\right) \\
& +J^{-2}\left(\mathbf{F} \mathbf{J}_{l}\right) \times\left(\mathbf{L F B} \mathbf{B}_{l}+\mathbf{F} \dot{\mathbf{B}}_{l}\right)-\mathbf{L}^{\mathrm{T}} \mathbf{F}^{-\mathrm{T}}\left[\operatorname{Grad}\left(J^{-1} \mathbf{F} \mathbf{B}_{l}\right)\right]^{\mathrm{T}}\left(\mathbf{F}^{-\mathrm{T}} \mathbf{M}_{l}\right) \\
& +\mathbf{F}^{-\mathrm{T}}\left[\operatorname{Grad}\left(-J^{-1}(\operatorname{div} \mathbf{u}) \mathbf{F} \mathbf{B}_{l}+J^{-1} \mathbf{L} \mathbf{F} \mathbf{B}_{l}+J^{-1} \mathbf{F} \dot{\mathbf{B}}_{l}\right)\right]^{\mathrm{T}}\left(\mathbf{F}^{-\mathrm{T}} \mathbf{M}_{l}\right) \\
& +\mathbf{F}^{-\mathrm{T}}\left[\operatorname{Grad}\left(J^{-1} \mathbf{F} \mathbf{B}_{l}\right)\right]^{\mathrm{T}}\left(-\mathbf{L}^{\mathrm{T}} \mathbf{F}^{-\mathrm{T}} \mathbf{M}_{l}+\mathbf{F}^{-\mathrm{T}} \dot{\mathbf{M}}_{l}\right) \\
& +\frac{\partial}{\partial t}\left[2 J^{-2}(\operatorname{div} \mathbf{u})\left(\mathbf{F} \mathbf{P}_{l}\right) \times\left(\mathbf{F B} \mathbf{B}_{l}\right)+J^{-2}\left(\mathbf{L F P} \mathbf{P}_{l}+\mathbf{F} \dot{\mathbf{P}}_{l}\right) \times\left(\mathbf{F} \mathbf{B}_{l}\right)\right. \\
& \left.+J^{-2}\left(\mathbf{F P}_{l}\right) \times\left(\mathbf{L F B} \mathbf{B}_{l}+\mathbf{F B}_{l}\right)\right]-J^{-1}(\operatorname{div} \mathbf{u}) \operatorname{Div}\left[J^{-1} \mathbf{V} \otimes\left\{\left(\mathbf{F P}_{l} \times\left(\mathbf{F B}_{l}\right)\right\}\right]\right.
\end{aligned}
$$




$$
\begin{aligned}
& +J^{-1} \operatorname{Div}\left[J^{-1} \dot{\mathbf{V}} \otimes\left\{\left(\mathbf{F P}_{l}\right) \times\left(\mathbf{F B}_{l}\right)\right\}-J^{-1}(\operatorname{div} \mathbf{u}) \mathbf{V} \otimes\left\{\left(\mathbf{F P}_{l}\right) \times\left(\mathbf{F B} \mathbf{B}_{l}\right)\right\}\right. \\
& \left.+J^{-1} \mathbf{V} \otimes\left\{\left(\mathbf{L F P} \mathbf{P}_{l}+\mathbf{F} \dot{\mathbf{P}}_{l}\right) \times\left(\mathbf{F} \mathbf{B}_{l}\right)+\left(\mathbf{F} \mathbf{P}_{l}\right) \times\left(\mathbf{L F B} \mathbf{B}_{l}+\mathbf{F} \dot{\mathbf{B}}_{l}\right)\right\}\right], \\
& \dot{\mathbf{L}}_{E}=-J^{-1}(\operatorname{div} \mathbf{u})\left\{\left(\mathbf{F} \mathbf{P}_{l}\right) \times\left(\mathbf{F}^{-\mathrm{T}} \mathbf{E}_{l}\right)+\left(\mathbf{F}^{-\mathrm{T}} \mathbf{M}_{e l}\right) \times\left(\mathbf{F B}_{l}\right)\right\} \\
& +J^{-1}\left(\mathbf{L F} \mathbf{P}_{l}+\mathbf{F} \dot{\mathbf{P}}_{l}\right) \times\left(\mathbf{F}^{-\mathrm{T}} \mathbf{E}_{l}\right)+J^{-1}\left(\mathbf{F} \mathbf{P}_{l}\right) \times\left(-\mathbf{L}^{\mathrm{T}} \mathbf{F}^{-\mathrm{T}} \mathbf{E}_{l}+\mathbf{F}^{-\mathrm{T}} \dot{\mathbf{E}}_{l}\right) \\
& +J^{-1}\left(\mathbf{F}^{-\mathrm{T}} \mathbf{M}_{e l}\right) \times\left(\mathbf{L} \mathbf{F} \mathbf{B}_{l}+\mathbf{F} \dot{\mathbf{B}}_{l}\right)+J^{-1}\left(-\mathbf{L}^{\mathrm{T}} \mathbf{F}^{-\mathrm{T}} \mathbf{M}_{e l}+\mathbf{F}^{-\mathrm{T}} \dot{\mathbf{M}}_{e l}\right) \times\left(\mathbf{F} \mathbf{B}_{l}\right) .
\end{aligned}
$$
become

When updated to Eulerian form, the balance equations (45) and (46)

$$
\begin{gathered}
\operatorname{div} \dot{\mathbf{T}}_{0}+(\operatorname{div} \mathbf{u}) \mathbf{f}_{e}+\dot{\mathbf{f}}_{E 0}=\rho \mathbf{u}_{t t}, \\
\boldsymbol{\varepsilon}\left(\mathbf{L} \boldsymbol{\tau}+\dot{\mathbf{T}}_{0}\right)+(\operatorname{div} \mathbf{u}) \mathbf{L}_{e}+\dot{\mathbf{L}}_{E 0}=\mathbf{0},
\end{gathered}
$$

where $\dot{\mathbf{f}}_{E 0}$ and $\dot{\mathbf{L}}_{E 0}$ are the push-forward forms of the incremental body force and moment, respectively, and are given by

$$
\begin{aligned}
& \dot{\mathbf{f}}_{E 0}=-(\operatorname{div} \mathbf{u}) \rho_{\mathrm{e}} \mathbf{E}+\dot{\rho}_{E 0} \mathbf{E}-\rho_{\mathrm{e}} \mathbf{L}^{\mathrm{T}} \mathbf{E}+\rho_{\mathrm{e}} \dot{\mathbf{E}}_{l 0}-\mathbf{L}^{\mathrm{T}}(\operatorname{grad} \mathbf{E})^{\mathrm{T}} \mathbf{P} \\
&+ {\left[\operatorname{grad}\left(-\mathbf{L}^{\mathrm{T}} \mathbf{E}+\dot{\mathbf{E}}_{l 0}\right)\right]^{\mathrm{T}} \mathbf{P}+(\operatorname{grad} \mathbf{E})^{\mathrm{T}}\left[-(\operatorname{div} \mathbf{u}) \mathbf{P}+\mathbf{L P}+\dot{\mathbf{P}}_{l 0}\right] } \\
&-2(\operatorname{div} \mathbf{u}) \mathbf{J} \times \mathbf{B}+\left(\mathbf{L J}+\dot{\mathbf{J}}_{l 0}\right) \times \mathbf{B}+\mathbf{J} \times\left(\mathbf{L B}+\dot{\mathbf{B}}_{l 0}\right)-\mathbf{L}^{\mathrm{T}}(\operatorname{grad} \mathbf{B})^{\mathrm{T}} \mathbf{M} \\
&+\left[\operatorname{grad}\left(-(\operatorname{div} \mathbf{u}) \mathbf{B}+\mathbf{L B}+\dot{\mathbf{B}}_{l 0}\right)\right]^{\mathrm{T}} \mathbf{M}+(\operatorname{grad} \mathbf{B})^{\mathrm{T}}\left(-\mathbf{L}^{\mathrm{T}} \mathbf{M}+\dot{\mathbf{M}}_{l 0}\right) \\
&+\frac{\partial}{\partial t}\left[2(\operatorname{div} \mathbf{u}) \mathbf{P} \times \mathbf{B}+\left(\mathbf{L P}+\dot{\mathbf{P}}_{l 0}\right) \times \mathbf{B}+\mathbf{P} \times\left(\mathbf{L B}+\dot{\mathbf{B}}_{l 0}\right)\right]
\end{aligned}
$$

$-(\operatorname{div} \mathbf{u}) \operatorname{div}[\mathbf{v} \otimes(\mathbf{P} \times \mathbf{B})]+\operatorname{div}[(\dot{\mathbf{v}}-\mathbf{L v}) \otimes(\mathbf{P} \times \mathbf{B})-(\operatorname{div} \mathbf{u}) \mathbf{v} \otimes(\mathbf{P} \times \mathbf{B})$

$$
\begin{gathered}
\left.+\mathbf{v} \otimes\left\{\left(\mathbf{L P}+\dot{\mathbf{P}}_{l 0}\right) \times \mathbf{B}\right\}+\mathbf{P} \times\left(\mathbf{L B}+\dot{\mathbf{B}}_{l 0}\right)\right], \\
\dot{\mathbf{L}}_{E 0}=-(\operatorname{div} \mathbf{u})\left(\mathbf{P} \times \mathbf{E}+\mathbf{M}_{e} \times \mathbf{B}\right)+\left(\mathbf{L P}+\dot{\mathbf{P}}_{l 0}\right) \times \mathbf{E}+\mathbf{P} \times\left(-\mathbf{L}^{\mathrm{T}} \mathbf{E}+\dot{\mathbf{E}}_{l 0}\right) \\
+\mathbf{M}_{e} \times\left(\mathbf{L B}+\dot{\mathbf{B}}_{l 0}\right)+\left(-\mathbf{L}^{\mathrm{T}} \mathbf{M}_{e}+\dot{\mathbf{M}}_{e l 0}\right) \times \mathbf{B} .
\end{gathered}
$$

The heat equation (27) can be incremented to give

$\operatorname{Div} \dot{\mathbf{q}}_{l}+\rho_{r} c_{p} \frac{\partial}{\partial t}\left[J^{-1} \dot{\vartheta}_{l}-J^{-1}(\operatorname{div} \mathbf{u}) \vartheta_{l}\right]=\dot{q}_{l}+\dot{w}_{E}+\dot{\mathbf{T}}: \operatorname{Grad}(\mathbf{F V})$

$$
+\mathbf{T}: \operatorname{Grad}(\mathbf{L F V}+\mathbf{F} \dot{\mathbf{V}}) \text {, }
$$


which when updated to the Eulerian configuration becomes

$$
\operatorname{div} \dot{\mathbf{q}}_{l 0}+\rho c_{p} \frac{\partial}{\partial t}\left[\dot{\vartheta}_{l 0}-(\operatorname{div} \mathbf{u}) \vartheta\right]=\dot{q}_{l 0}+\dot{w}_{E 0}+\dot{\mathbf{T}}_{0}: \operatorname{grad} \mathbf{v}+\tau: \operatorname{grad} \dot{\mathbf{v}}
$$

We have used the push-forward relations $\dot{\mathbf{q}}_{l 0}=J^{-1} \mathbf{F} \dot{\mathbf{q}}_{l}, \dot{\vartheta}_{l 0}=J^{-1} \dot{\vartheta}_{l}, \dot{w}_{E 0}=$ $J^{-1} \dot{w}_{E}$, and $\dot{q}_{l 0}=J^{-1} \dot{q}_{l}$ to effect the above transformation. The increment in the electromagnetic power is given by

$$
\begin{aligned}
& \dot{w}_{E}=\left(\mathbf{L} \mathbf{F} \mathbf{J}_{e l}+\mathbf{F} \dot{\mathbf{J}}_{e l}\right) \cdot\left(\mathbf{F}^{-\mathrm{T}} \mathbf{E}_{e l}\right)+\left(\mathbf{F} \mathbf{J}_{e l}\right) \cdot\left(-\mathbf{L}^{\mathrm{T}} \mathbf{F}^{-\mathrm{T}} \mathbf{E}_{e l}+\mathbf{F}^{-\mathrm{T}} \cdot \dot{\mathbf{E}}_{e l}\right) \\
& +\rho_{r}\left[\frac{\partial}{\partial t}\left(\frac{\mathbf{L F P}}{\rho_{r}+\mathbf{F} \dot{\mathbf{P}}_{l}}\right)+\operatorname{Grad}\left(\frac{\mathbf{L F P}}{\rho_{r}+\mathbf{F} \dot{\mathbf{P}}_{l}}\right) \mathbf{V}+\operatorname{Grad}\left(\frac{\mathbf{F P}}{\rho_{r}}\right) \dot{\mathbf{V}}\right] \\
& \cdot\left(\mathbf{F}^{-\mathrm{T}} \mathbf{E}_{e l}\right)+\rho_{r}\left[\frac{\partial}{\partial t}\left(\frac{\mathbf{F} \mathbf{P}_{l}}{\rho_{r}}\right)+\operatorname{Grad}\left(\frac{\mathbf{F} \mathbf{P}_{l}}{\rho_{r}}\right) \mathbf{V}\right] \cdot\left(-\mathbf{L}^{\mathrm{T}} \mathbf{F}^{-\mathrm{T}} \mathbf{E}_{e l}+\mathbf{F}^{-\mathrm{T}} \cdot \dot{\mathbf{E}}_{e l}\right) \\
& -J\left((\operatorname{div} \mathbf{u}) \mathbf{F}^{-\mathrm{T}} \mathbf{M}_{e l}-\mathbf{L}^{\mathrm{T}} \mathbf{F}^{-\mathrm{T}} \mathbf{M}_{e l}+\mathbf{F}^{-\mathrm{T}} \dot{\mathbf{M}}_{e l}\right) \cdot\left[\frac{\partial}{\partial t}\left(J^{-1} \mathbf{F} \mathbf{B}_{l}\right)\right. \\
& \left.+\operatorname{Grad}\left(J^{-1} \mathbf{F B}_{l}\right) \mathbf{V}\right]-J \mathbf{F}^{-\mathrm{T}} \mathbf{M}_{e l} \cdot\left[\frac{\partial}{\partial t} J^{-1}\left(-(\operatorname{div} \mathbf{u}) \mathbf{F} \mathbf{B}_{l}+\mathbf{L F B} \mathbf{B}_{l}+\mathbf{F} \dot{\mathbf{B}}_{l}\right)\right. \\
& \left.+\operatorname{Grad} J^{-1}\left\{-(\operatorname{div} \mathbf{u}) \mathbf{F} \mathbf{B}_{l}+\mathbf{L F B} \mathbf{B}_{l}+\mathbf{F} \dot{\mathbf{B}}_{l}\right\} \mathbf{V}+\operatorname{Grad}\left(J^{-1} \mathbf{F} \mathbf{B}_{l}\right) \dot{\mathbf{V}}\right] .
\end{aligned}
$$

This is given in Eulerian form as

$$
\begin{array}{r}
\dot{w}_{E 0}=\left(\mathbf{L} \mathbf{J}_{e}+\dot{\mathbf{J}}_{e l 0}\right) \cdot \mathbf{E}_{e}+\mathbf{J}_{e} \cdot\left(-\mathbf{L}^{\mathrm{T}} \mathbf{E}_{e}+\dot{\mathbf{E}}_{e l 0}\right) \\
\rho\left[\frac{d}{d t}\left(\frac{\mathbf{L P}+\dot{\mathbf{P}}_{l 0}}{\rho}\right)+\operatorname{grad}\left(\frac{\mathbf{P}}{\rho}\right)(\dot{\mathbf{v}}-\mathbf{L v})\right] \cdot \mathbf{E}_{e}+\rho \frac{d}{d t}\left(\frac{\mathbf{P}}{\rho}\right) \cdot\left(-\mathbf{L}^{\mathrm{T}} \mathbf{E}_{e}+\dot{\mathbf{E}}_{e l 0}\right) \\
-\left\{(\operatorname{div} \mathbf{u}) \mathbf{M}_{e}-\mathbf{L}^{\mathrm{T}} \mathbf{M}_{e}+\dot{\mathbf{M}}_{e l 0}\right\} \cdot \frac{d \mathbf{B}}{d t}-\mathbf{M}_{e} \cdot\left[\frac{d}{d t}\left\{-(\operatorname{div} \mathbf{u}) \mathbf{B}+\mathbf{L B}+\dot{\mathbf{B}}_{l 0}\right\}\right. \\
+(\operatorname{grad} \mathbf{B})(\dot{\mathbf{v}}-\mathbf{L v})] .
\end{array}
$$

\subsection{Incremental constitutive equations and Moduli tensors}

On incrementing the constitutive equations (30), we obtain

$$
\dot{\mathbf{T}}=\mathcal{A} \dot{\mathbf{F}}+\mathcal{B} \dot{\mathbf{E}}_{e l}+\mathcal{C} \dot{\mathbf{B}}_{l}+\mathcal{D} \dot{\vartheta}_{l}, \quad \dot{\mathbf{P}}_{l}=-\left(\mathcal{F} \dot{\mathbf{F}}+\mathcal{G} \dot{\mathbf{E}}_{e l}+\mathcal{H} \dot{\mathbf{B}}_{l}+\mathcal{I} \dot{\vartheta}_{l}\right),
$$

and

$$
\dot{\mathbf{M}}_{e l}=-\left(\mathcal{K} \dot{\mathbf{F}}+\mathcal{L} \dot{\mathbf{E}}_{e l}+\mathcal{M} \dot{\mathbf{B}}_{l}+\mathcal{N} \dot{\vartheta}_{l}\right)
$$


where we have defined the moduli tensors as

$$
\begin{aligned}
& \mathcal{A}=\frac{\partial^{2} \Phi}{\partial \mathbf{F} \partial \mathbf{F}}, \quad \mathcal{B}=\frac{\partial^{2} \Phi}{\partial \mathbf{E}_{e l} \partial \mathbf{F}}, \quad \mathcal{C}=\frac{\partial^{2} \Phi}{\partial \mathbf{B}_{l} \partial \mathbf{F}}, \mathcal{D}=\frac{\partial^{2} \Phi}{\partial \vartheta_{l} \partial \mathbf{F}} \\
& \mathcal{F}=\frac{\partial^{2} \Phi}{\partial \mathbf{F} \partial \mathbf{E}_{e l}}, \quad \mathcal{G}=\frac{\partial^{2} \Phi}{\partial \mathbf{E}_{e l} \partial \mathbf{E}_{e l}}, \quad \mathcal{H}=\frac{\partial^{2} \Phi}{\partial \mathbf{B}_{l} \partial \mathbf{E}_{e l}}, \mathcal{I}=\frac{\partial^{2} \Phi}{\partial \vartheta_{l} \partial \mathbf{E}_{e l}} \\
& \mathcal{K}=\frac{\partial^{2} \Phi}{\partial \mathbf{F} \partial \mathbf{B}_{l}}, \quad \mathcal{L}=\frac{\partial^{2} \Phi}{\partial \mathbf{E}_{e l} \partial \mathbf{B}_{l}}, \quad \mathcal{M}=\frac{\partial^{2} \Phi}{\partial \mathbf{B}_{l} \partial \mathbf{B}_{l}}, \quad \mathcal{N}=\frac{\partial^{2} \Phi}{\partial \vartheta_{l} \partial \mathbf{B}_{l}}
\end{aligned}
$$

Here $\mathcal{A}$ is a fourth-order tensor, $\mathcal{B}, \mathcal{C}, \mathcal{F}, \mathcal{K}$ are third-order tensors, $\mathcal{G}, \mathcal{H}, \mathcal{L}, \mathcal{M}$ are second-order tensors, and $\mathcal{D}, \mathcal{I}, \mathcal{N}$ are first-order tensors (or vectors). To put them in perspective, for a problem involving simpler quasimagnetostatic case as presented in [11] and [14], only $\mathcal{A}, \boldsymbol{C}$, and $\mathcal{M}$ are required. Products in (57) and (58) are defined in component form as

$$
\begin{aligned}
& (\mathcal{A} \dot{\mathbf{F}})_{\alpha i}=\mathcal{A}_{\alpha i \beta j} \dot{F}_{j \beta}, \quad\left(\mathcal{B} \dot{\mathbf{E}}_{e l}\right)_{\alpha i}=\mathcal{B}_{\alpha i \mid \beta} \dot{E}_{e l \beta}, \quad\left(C \dot{\mathbf{B}}_{l}\right)_{\alpha i}=C_{\alpha i \mid \beta} \dot{B}_{l \beta}, \\
& (\mathcal{F} \dot{\mathbf{F}})_{i}=\mathcal{F}_{i \mid \alpha j} \dot{F}_{j \alpha}, \quad\left(\mathcal{G} \dot{\mathbf{E}}_{e l}\right)_{\alpha}=\mathcal{G}_{\alpha \beta} \dot{E}_{e l \beta}, \quad\left(\mathcal{H} \dot{\mathbf{B}}_{l}\right)_{\alpha}=\mathcal{H}_{\alpha \beta} \dot{B}_{l \beta}, \\
& (\mathcal{K} \dot{\mathbf{F}})_{i}=\mathcal{K}_{i \mid \alpha j} \dot{F}_{j \alpha}, \quad\left(\mathcal{L} \dot{\mathbf{E}}_{e l}\right)_{\alpha}=\mathcal{L}_{\alpha \beta} \dot{E}_{e l \beta}, \quad\left(\mathcal{M} \dot{\mathbf{B}}_{l}\right)_{\alpha}=\mathcal{M}_{\alpha \beta} \dot{B}_{l \beta},
\end{aligned}
$$

and the following relations hold

$$
\mathcal{K}=C^{\mathrm{T}}, \quad \mathcal{F}=\mathcal{B}^{\mathrm{T}}, \quad \mathcal{L}=\mathcal{H}^{\mathrm{T}} .
$$

On updating (push-forward to Eulerian configuration) the incremented constitutive equations (57) and (58), we obtain

$$
\begin{gathered}
\dot{\mathbf{T}}_{0}=\mathcal{A}_{0} \mathbf{L}+\mathcal{B}_{0} \dot{\mathbf{E}}_{e l 0}+\mathcal{C}_{0} \dot{\mathbf{B}}_{l 0}+\mathcal{D}_{0} \dot{\vartheta}_{l 0}, \\
\dot{\mathbf{P}}_{l 0}=-\left(\mathcal{B}_{0}^{\mathrm{T}} \mathbf{L}+\mathcal{G}_{0} \dot{\mathbf{E}}_{e l 0}+\mathcal{H}_{0} \dot{\mathbf{B}}_{l 0}+\mathcal{I}_{0} \dot{\vartheta}_{l 0}\right),
\end{gathered}
$$

and

$$
\dot{\mathbf{M}}_{e l 0}=-\left(\boldsymbol{C}_{0}^{\mathrm{T}} \mathbf{L}+\mathcal{H}_{0}^{\mathrm{T}} \dot{\mathbf{E}}_{e l 0}+\mathcal{M}_{0} \dot{\mathbf{B}}_{l 0}+\mathcal{N}_{0} \dot{\vartheta}_{l 0}\right),
$$

with $\dot{\vartheta}_{l 0}=J \dot{\vartheta}_{l}$ and the updated moduli tensors defined in component form as

$$
\begin{array}{r}
\mathcal{A}_{0 p i q j}=J^{-1} F_{p \alpha} F_{q \beta} \mathcal{A}_{\alpha i \beta j}, \quad \mathcal{B}_{0 i j \mid k}=J^{-1} F_{i \alpha} F_{k \beta} \mathcal{B}_{\alpha j \mid \beta}, \quad \mathcal{C}_{0 i j \mid k}=F_{i \alpha} F_{\beta k}^{-1} \mathcal{C}_{\alpha j \mid \beta}, \\
\mathcal{D}_{0 i j}=F_{i k} \mathcal{D}_{k j}, \quad \mathcal{G}_{0 i j}=J^{-1} F_{i \alpha} F_{j \beta} \mathcal{G}_{\alpha \beta}, \quad \mathcal{H}_{0 i j}=F_{i \alpha} F_{\beta j}^{-1} \mathcal{H}_{\alpha \beta}, \\
\mathcal{I}_{0 i}=F_{i k} \mathcal{I}_{k}, \quad \mathcal{M}_{0 i j}=J F_{\alpha i}^{-1} F_{\beta j}^{-1} \mathcal{M}_{\alpha \beta}, \quad \mathcal{N}_{0 i}=J^{-1} F_{k i}^{-1} \mathcal{N}_{k} .
\end{array}
$$

Incrementing and updating the constitutive equations (34), we get

$$
\dot{\mathbf{q}}_{l 0}=-(\operatorname{div} \mathbf{u}) \kappa \operatorname{grad} \vartheta+2 \mathbf{L} \kappa \operatorname{grad} \vartheta-\kappa \operatorname{grad}\left[\dot{\vartheta}_{l 0}-(\operatorname{div} \mathbf{u}) \vartheta\right],
$$


and

$$
\dot{\mathbf{J}}_{l 0}=\boldsymbol{\xi}\left[\left\{(\operatorname{div} \mathbf{u}) \mathbf{I}-\mathbf{L}-\mathbf{L}^{\mathrm{T}}\right\} \mathbf{E}+\dot{\mathbf{E}}_{l 0}\right] .
$$

On substituting the incremented updated constitutive equations (62), (63), (64), (66), and (67) into the incremented updated balance equations (40), (41), (49), (50), and (54), we obtain

$$
\begin{aligned}
& \varepsilon_{0} \operatorname{div}\left[\dot{\mathbf{E}}_{l 0}+\left\{(\operatorname{div} \mathbf{u}) \mathbf{I}-\left(\mathbf{L}+\mathbf{L}^{\mathrm{T}}\right)\right\} \mathbf{E}\right]=\dot{\rho}_{E 0} \\
& -\operatorname{div}\left(\mathcal{B}_{0}^{\mathrm{T}} \mathbf{L}+\mathcal{G}_{0} \dot{\mathbf{E}}_{e l 0}+\mathcal{H}_{0} \dot{\mathbf{B}}_{l 0}+\mathcal{I}_{0} \dot{\vartheta}_{l 0}\right), \\
& \mu_{0}^{-1} \operatorname{curl}\left[\{(1+\operatorname{div} \mathbf{u}) \mathbf{I}+2 \mathbf{L}\} \dot{\mathbf{B}}_{l 0}\right]-\varepsilon_{0} \operatorname{curl}\left(\mathbf{u}_{, t} \times \mathbf{E}+\mathbf{v} \times \hat{\mathbf{E}}\right)-\varepsilon_{0} \hat{\mathbf{E}}_{, t} \\
& =-\operatorname{curl}\left(\boldsymbol{C}_{0}^{\mathrm{T}} \mathbf{L}+\boldsymbol{H}_{0}^{\mathrm{T}} \dot{\mathbf{E}}_{e l 0}+\mathcal{M}_{0} \dot{\mathbf{B}}_{l 0}+\mathcal{N}_{0} \dot{\vartheta}_{l 0}\right)+\dot{\mathbf{J}}_{\mathrm{E} 0} \\
& -[(\operatorname{div} \mathbf{u}) \mathbf{I}-\boldsymbol{\Gamma}]\left(\mathcal{B}_{0}^{\mathrm{T}} \mathbf{L}+\mathcal{G}_{0} \dot{\mathbf{E}}_{e l 0}+\mathcal{H}_{0} \dot{\mathbf{B}}_{l 0}+\mathcal{I}_{0} \dot{\vartheta}_{l 0}\right) \\
& -\left(\mathcal{B}_{0}^{\mathrm{T}} \mathbf{L}+\mathcal{G}_{0} \dot{\mathbf{E}}_{e l 0}+\mathcal{H}_{0} \dot{\mathbf{B}}_{l 0}+\mathcal{I}_{0} \dot{\vartheta}_{l 0}\right)_{, t}, \\
& \operatorname{div}\left(\mathcal{A}_{0} \mathbf{L}+\mathcal{B}_{0} \dot{\mathbf{E}}_{e l 0}+\boldsymbol{C}_{0} \dot{\mathbf{B}}_{l 0}+\mathcal{D}_{0} \dot{\vartheta}_{l 0}\right)+(\operatorname{div} \mathbf{u}) \mathbf{f}_{e}+\dot{\mathbf{f}}_{E 0}=\rho \mathbf{u}_{, t}, \\
& \boldsymbol{\varepsilon}\left(\mathbf{L} \tau+\mathcal{A}_{0} \mathbf{L}+\mathcal{B}_{0} \dot{\mathbf{E}}_{e l 0}+\boldsymbol{C}_{0} \dot{\mathbf{B}}_{l 0}+\mathcal{D}_{0} \dot{\vartheta}_{l 0}\right)+(\operatorname{div} \mathbf{u}) \mathbf{L}_{e}+\dot{\mathbf{L}}_{E 0}=\mathbf{0}, \\
& \operatorname{div}\left[-(\operatorname{div} \mathbf{u}) \kappa \operatorname{grad} \vartheta+2 \mathbf{L} \kappa \operatorname{grad} \vartheta-\kappa \operatorname{grad}\left(\dot{\vartheta}_{l 0}-(\operatorname{div} \mathbf{u}) \vartheta\right)\right] \\
& =-\rho c_{p} \frac{\partial}{\partial t}\left[\dot{\vartheta}_{l 0}-(\operatorname{div} \mathbf{u}) \vartheta\right]+\dot{q}_{l 0}+\dot{w}_{E 0}+\boldsymbol{\tau}: \operatorname{grad} \mathbf{u}_{, t} \\
& +\left(\mathcal{A}_{0} \mathbf{L}+\mathcal{B}_{0} \dot{\mathbf{E}}_{e l 0}+\mathcal{C}_{0} \dot{\mathbf{B}}_{l 0}+\mathcal{D}_{0} \dot{\vartheta}_{l 0}\right): \operatorname{grad} \mathbf{v},
\end{aligned}
$$

along with (39). The governing equations of the incremental fields in the region $\mathcal{P}$ are

$$
\operatorname{div} \dot{\mathbf{B}}=0, \quad \varepsilon_{r} \operatorname{div} \dot{\mathbf{E}}=0, \quad \operatorname{curl} \dot{\mathbf{E}}=-\frac{\partial \dot{\mathbf{B}}}{\partial t}, \quad \frac{1}{\mu_{0} \mu_{r}} \operatorname{curl} \dot{\mathbf{B}}=\dot{\mathbf{J}}+\varepsilon_{0} \varepsilon_{r} \frac{\partial \dot{\mathbf{E}}}{\partial t},
$$

while in vacuum, we have

$$
\operatorname{div} \dot{\mathbf{B}}^{*}=0, \quad \operatorname{div} \dot{\mathbf{E}}^{*}=0, \quad \operatorname{curl} \dot{\mathbf{E}}^{*}=-\frac{\partial \dot{\mathbf{B}}^{*}}{\partial t}, \quad \operatorname{curl} \dot{\mathbf{B}}^{*}=\varepsilon_{0} \mu_{0} \frac{\partial \dot{\mathbf{E}}^{*}}{\partial t}
$$

\subsection{Incremental boundary conditions}

At the boundary $\partial \mathcal{B}_{r}$ of the continuum and vacuum, the incremental form of the boundary conditions (10)-(13) is given as

$$
\mathbf{N} \times\left(\dot{\mathbf{E}}_{l}+\dot{\mathbf{V}} \times \mathbf{B}_{l}+\mathbf{V} \times \dot{\mathbf{B}}_{l}-\mathbf{F}^{\mathrm{T}} \mathbf{L} \mathbf{E}^{*}-\mathbf{F}^{\mathrm{T}} \dot{\mathbf{E}}^{*}\right)=\mathbf{0},
$$




$$
\begin{array}{r}
\mathbf{N} \cdot\left(\dot{\mathbf{B}}_{l}-J(\operatorname{div} \mathbf{u}) \mathbf{F}^{-1} \mathbf{B}^{*}-J \mathbf{F}^{-1} \mathbf{L}^{-1} \mathbf{B}^{*}-J \mathbf{F}^{-1} \dot{\mathbf{B}}^{*}\right)=0, \\
\mathbf{N} \cdot\left\{\varepsilon_{0} J \mathbf{c}^{-1}\left(\dot{\mathbf{E}}_{l}-\mathbf{F}^{\mathrm{T}} \mathbf{L}^{\mathrm{T}} \mathbf{E}^{*}-\mathbf{F}^{\mathrm{T}} \dot{\mathbf{E}}^{*}\right)+\varepsilon_{0} J(\operatorname{div} \mathbf{u}) \mathbf{c}^{-1}\left(\mathbf{E}_{l}-\mathbf{F}^{\mathrm{T}} \mathbf{E}^{*}\right)\right. \\
\left.+\varepsilon_{0} J\left(\mathbf{F}^{-1} \mathbf{L}^{-1} \mathbf{F}^{-\mathrm{T}}+\mathbf{F}^{-1} \mathbf{L}^{-\mathrm{T}} \mathbf{F}^{-\mathrm{T}}\right)\left(\mathbf{E}_{l}-\mathbf{F}^{\mathrm{T}} \mathbf{E}^{*}\right)+\dot{\mathbf{P}}_{l}\right\}=\dot{\sigma}_{E}, \\
\mathbf{N} \times\left\{J^{-1} \mu_{0}^{-1} \dot{\mathbf{B}}_{l}-J^{-1}(\operatorname{div} \mathbf{u}) \mu_{0}^{-1} \mathbf{c} \mathbf{B}_{l}+J^{-1} \mu_{0}^{-1}\left(\mathbf{F}^{\mathrm{T}} \mathbf{L} \mathbf{F}+\mathbf{F}^{\mathrm{T}} \mathbf{L}^{\mathrm{T}} \mathbf{F}\right) \mathbf{B}_{l}\right. \\
-\dot{\mathbf{M}}_{l}-\dot{\mathbf{V}} \times\left(\varepsilon_{0} J \mathbf{c}^{-1} \mathbf{E}_{l}+\mathbf{P}_{l}\right)-\varepsilon_{0} \mathbf{V} \times\left(J(\operatorname{div} \mathbf{u}) \mathbf{c}^{-1} \mathbf{E}_{l}+J \mathbf{c}^{-1} \dot{\mathbf{E}}_{l}\right. \\
\left.\left.+J\left(\mathbf{F}^{-1} \mathbf{L}^{-1} \mathbf{F}^{-\mathrm{T}}+\mathbf{F}^{-1} \mathbf{L}^{-\mathrm{T}} \mathbf{F}^{-\mathrm{T}}\right) \mathbf{E}_{l}+\dot{\mathbf{P}}_{l}\right)-\mu_{0}^{-1} \mathbf{F}^{\mathrm{T}} \mathbf{L}^{\mathrm{T}} \mathbf{B}^{*}-\mu_{0}^{-1} \mathbf{F}^{\mathrm{T}} \dot{\mathbf{B}}^{*}\right\} \\
=\dot{\mathbf{K}}_{l}-\dot{\sigma}_{E} \mathbf{V}_{\mathrm{s}}-\sigma_{E} \dot{\mathbf{V}}_{\mathrm{s}} .
\end{array}
$$

We recollect the Nanson's formula $\mathbf{n} d a=J \mathbf{F}^{-\mathrm{T}} \mathbf{N} d A$ connecting reference and current area elements $d A$ and $d a$, where $\mathbf{n}$ is the unit outward normal to $\partial \mathcal{B}_{t}$. The surface current and electric charge densities in the two configurations are related by $\mathbf{K}_{l}=\mathbf{F}^{-1} \mathbf{K} d a / d A$ and $\sigma_{E}=\sigma_{e} d a / d A$. Using these relations and the relations (38), we rewrite the above incremental boundary conditions in the current configuration on $\partial \mathcal{B}_{t}$ as

$$
\begin{gathered}
\mathbf{n} \times\left(\dot{\mathbf{E}}_{l 0}+(\dot{\mathbf{v}}-\mathbf{L v}) \times \mathbf{B}+\mathbf{v} \times \dot{\mathbf{B}}_{l 0}-\mathbf{L} \mathbf{E}^{*}-\dot{\mathbf{E}}^{*}\right)=\mathbf{0} \\
\mathbf{n} \cdot\left(\dot{\mathbf{B}}_{l 0}-(\operatorname{div} \mathbf{u}) \mathbf{B}^{*}-\mathbf{L}^{-1} \mathbf{B}^{*}-\dot{\mathbf{B}}^{*}\right)=0 \\
\mathbf{n} \cdot\left\{\varepsilon_{0}\left(\dot{\mathbf{E}}_{l 0}-\mathbf{L}^{\mathrm{T}} \mathbf{E}^{*}-\dot{\mathbf{E}}^{*}\right)+\varepsilon_{0}(\operatorname{div} \mathbf{u})\left(\mathbf{E}-\mathbf{E}^{*}\right)+\dot{\mathbf{P}}_{l 0}\right. \\
+\varepsilon_{0}\left(\mathbf{L}^{-1}+\mathbf{L}^{-\mathrm{T}}\right)\left(\mathbf{E}-\mathbf{E}^{*}\right)=\dot{\sigma}_{E 0}, \\
\mathbf{n} \times\left\{\mu_{0}^{-1} \dot{\mathbf{B}}_{l 0}-\mu_{0}^{-1}(\operatorname{div} \mathbf{u}) \mathbf{B}+\mu_{0}^{-1}\left(\mathbf{L}+\mathbf{L}^{\mathrm{T}}\right) \mathbf{B}-\dot{\mathbf{M}}_{l 0}-(\dot{\mathbf{v}}-\mathbf{L v}) \times\left(\varepsilon_{0} \mathbf{E}+\mathbf{P}\right)\right. \\
\left.-\varepsilon_{0} \mathbf{v} \times\left((\operatorname{div} \mathbf{u}) \mathbf{E}+\dot{\mathbf{E}}_{l 0}+\left(\mathbf{L}^{-1}+\mathbf{L}^{-\mathrm{T}}\right) \mathbf{E}+\dot{\mathbf{P}}_{l 0}\right)-\mu_{0}^{-1} \mathbf{L}^{\mathrm{T}} \mathbf{B}^{*}-\mu_{0}^{-1} \dot{\mathbf{B}}^{*}\right\} \\
=\dot{\mathbf{K}}_{l 0}+\mathbf{L}^{-1} \mathbf{K}+\sigma_{e} \mathbf{L} \mathbf{v}_{\mathrm{s}},
\end{gathered}
$$

where we have defined $\dot{\mathbf{K}}_{l 0}=\mathbf{F} \dot{\mathbf{K}}_{l} d A / d a$ and $\dot{\sigma}_{E 0}=\dot{\sigma}_{E} d A / d a$.

At the boundary $\partial \mathcal{P}$, incremental form of the boundary conditions (14) is given as

$$
\begin{aligned}
\mathbf{n} \times\left(\dot{\mathbf{E}}-\dot{\mathbf{E}}^{*}\right) & =\mathbf{0}, \quad \mathbf{n} \cdot\left(\varepsilon_{r} \dot{\mathbf{E}}-\dot{\mathbf{E}}^{*}\right)=0, \\
\mathbf{n} \times\left(\frac{\dot{\mathbf{B}}}{\mu_{r}}-\dot{\mathbf{B}}^{*}\right) & =\mathbf{0}, \quad \mathbf{n} \cdot\left(\dot{\mathbf{B}}-\dot{\mathbf{B}}^{*}\right)=0
\end{aligned}
$$


Thus, a generic EMAT problem requires the solution of equations (39) and (68)-(72) in $\mathcal{B}_{t}$, equations (73) in $\mathcal{P}$, and equations (74) in vacuum using the boundary conditions (79) $-(82)$ at $\partial \mathcal{B}_{t}$ and (83) at $\partial \mathcal{P}$.

\section{Concluding remarks}

This paper reviews the existing theory of electromagnetic interactions with a solid continuum. In particular, we analyze the problem of wave propagation in a finitely deformed elastic solid with an underlying electric, magnetic, and temperature field. Unlike the existing literature, we generalize a nonlinear theory of elasticity to include electromagnetic effects. On a finite deformation, magnetic, and electric field, the equations are linearized to consider wave propagation and several moduli tensors are introduced. In addition to filling a gap in literature, it is expected that this work will lead to an accurate mathematical modelling of the 'Electromagnetic acoustic transducers' in particular.

\section{Acknowledgements}

This work was undertaken when the author was at the University of Glasgow supported by a university postgraduate scholarship and a UK ORS scholarship. Thanks are also extended to Prof. Ray W. Ogden, FRS of the University of Glasgow, UK for his helpful guidance during the initial phase of this work.

Manuscript received by Editorial Board, November 29, 2012; final version, March 17, 2013.

\section{REFERENCES}

[1] Hirao M., Ogi H.: EMATs for Science and Industry: Noncontacting Ultrasonic Measurements, Kluwer Academic Publishers, 2003.

[2] Ludwig R., You Z., Palanisamy R.: Numerical simulations of an electromagnetic acoustic transducer-receiver system for NDT applications, IEEE Transactions on Magnetics 29(3) (1993) 2081-2089.

[3] Ogi H.: Field dependence of coupling efficiency between electromagnetic field and ultrasonic bulk waves, Journal of Applied Physics 82(8) (1997) 3940.

[4] Thompson R. B.: A Model for the Electromagnetic Generation of Ultrasonic Guided Waves in Ferromagnetic Metal Polycrystals, IEEE Transactions on Sonics and Ultrasonics 25(1) (1978) 7-15.

[5] Shapoorabadi R. J., Konrad A., Sinclair A. N.: The governing electrodynamic equations of electromagnetic acoustic transducers, Journal of Applied Physics 97(10) (2005) 6-8.

[6] Pao Y. H.: Electromagnetic forces in deformable continua, in: S. Nemat-Nasser (Ed.), Mechanics Today, Vol. 4, Oxford University Press, 1978, pp. 209-305.

[7] Eringen A. C., Maugin G. A.: Electrodynamics of Continua, Vol. 1, Springer-Verlag, 1990. 
[8] Maugin G. A.: On modelling electromagnetomechanical interactions in deformable solids, International Journal of Advances in Engineering Sciences and Applied Mathematics 1 (2009) 25-32.

[9] Jolly M. R., Carlson J. D., Muñoz B. C.: A model of the behaviour of magnetorheological materials, Smart Materials and Structures 5(5) (1996) 607-614.

[10] Böse H., Rabindranath R., Ehrlich J.: Soft magnetorheological elastomers as new actuators for valves, Journal of Intelligent Material Systems and Structures 23(9) (2012) 989-994.

[11] Dorfmann A., Ogden R. W.: Nonlinear magnetoelastic deformations, Quarterly Journal of Mechanics and Applied Mathematics 57(7) (2004) 599-622.

[12] Dorfmann A., Ogden R. W.: Nonlinear electroelastic deformations, Journal of Elasticity 82(2) (2006) 99-127.

[13] Destrade M., Ogden R. W.: On magneto-acoustic waves in finitely deformed elastic solids, Mathematics and Mechanics of Solids 16(6) (2011) 594-604.

[14] Saxena P., Ogden R. W.: On surface waves in a finitely deformed magnetoelastic half-space, International Journal of Applied Mechanics 3(4) (2011) 633-665.

[15] Saxena P.: On Wave Propagation in Finitely Deformed Magnetoelastic Solids, Ph.D. thesis, University of Glasgow (2012).

[16] Coleman B. D., Noll W.: The thermodynamics of elastic materials with heat conduction and viscosity, Archive for Rational Mechanics and Analysis 13(1) (1963) 167-178.

\section{Ogólne równania opisujące działanie przetworników elektromagnetyczno-akustycznych}

\section{Streszczenie}

W artykule przedstawiono ogólne równania elektrodynamiki i mechaniki kontinuum, które uwzględnia się przy modelowaniu matematycznym właściwości przetworników elektromagnetycznoakustycznych (EMAT). Rozpatrzono istnienie skończonych deformacji w materiałach miękkich oraz możliwość powstawania prądów elektrycznych, gradientów temperatury i wewnętrznego wydzielania ciepła w wyniku rozpraszania energii. Punktem wyjścia były równania Maxwella i prawa równowagi nieliniowej teorii sprężystości. Na tej podstawie wyprowadzono równania i warunki brzegowe $\mathrm{w}$ formie przyrostowej, co umożliwiło rozwiązanie problemu propagacji fali o typie odpowiadającym wartościom granicznym. 